\title{
EFEITO de CONCENTRAÇões DE HERBICIDAS SOBRe ASPECTOS BIOLÓGICOS DE Fusarium SP. (ISOLADO FCAV\# 940) ${ }^{1}$
}

\author{
Effect of Herbicide Concentrations on Biological Aspects of Fusarium sp. \\ (Isolation FCAV\#940)
}

MENDES, D. ${ }^{2}$, PITELLI, R.A. ${ }^{3}$ e COELHO, L. ${ }^{4}$

\begin{abstract}
RESUMO - O fungo Fusarium sp. (isolado FCAV\#940) tem potencial de controle biológico das macrófitas aquáticas, Egeria najase Egeria densa, plantas que causam prejuízos de diferentes naturezas em ambientes lacustres. Com o objetivo de avaliar a associação desse fungo com o controle químico, visando otimizar o controle, foi avaliado o efeito de concentrações de herbicidas sobre o crescimento micelial, esporulação e germinação de macroconídios de Fusarium sp. (isolado FCAV\#940). As doses utilizadas para o cálculo das concentrações dos herbicidas foram: 1/2, 1,0 e 2,0 X a dose recomendada pelo fabricante. Por se tratar de macrófitas aquáticas submersas, foi considerada uma concentração de volume diluído, ou seja, a utilização da dose desejada, porém diluída em uma lâmina d'água de $15 \mathrm{~cm}$. Os herbicidas e as concentrações de i.a. avaliadas foram: triclopyr a 6,0, 3,0 e 1,5 $\mathrm{mg} \mathrm{L}^{-1}$; glyphosate a 6,87, 3,44 e $1,72 \mathrm{mg} \mathrm{L}^{-1}$; 2,4-D a 8,54, 4,27 e 2,14 $\mathrm{mg} \mathrm{L}^{-1}$; diquat a 2,0, 1,0 e 0,5 $\mathrm{mg} \mathrm{L}^{-1}$; fluridone a 200, 100 e $50 \mu \mathrm{gL}^{-1}$; e a testemunha (BDA). Os herbicidas e as concentrações testadas não apresentaram efeito inibitório no crescimento micelial, na esporulação e na germinação dos macroconídios do fungo.
\end{abstract}

Palavras-chave : crescimento micelial, esporulação, germinação, fungo e inibição.

\begin{abstract}
Fusarium sp. (isolation FCAV\# 940) has potential for the biological control of the macrophytas Egeria najas and Egeria densa, plants that cause losses of several kinds in water bodies. This study evaluated the likelihood of associating the fungus to concentrations of herbicides to optimize weed control, by measuring the inhibitory effects of several concentrations of herbicides on Fusarium sp. mycelial growth, sporulation and spore germination. The herbicide concentrations analyzed were: $1 / 2,1.0$ and 2.0 the concentration recommended by the manufacturer. Since these aquatic weeds are submerged, a diluted concentration in $15 \mathrm{~cm}$ water depth was considered. The herbicides and concentrations (a.i.) evaluated were: triclopyr at 6.0; 3.0 and $1.5 \mathrm{mg} \mathrm{L}^{-1}$; glyphosate at 6.87; 3.44 and $1.72 \mathrm{mg} \mathrm{L}^{-1}$; 2.4-D at 8.54; 4.27 and $2.14 \mathrm{mg} \mathrm{L}^{-1}$; diquat at 2.0; 1.0 and $0.5 \mathrm{mg} \mathrm{L}^{-1}$; fluridone at 200; 100 and $50 \mu \mathrm{g} \mathrm{L} \mathrm{L}^{-1}$; and a control (non-amended PDA). The results indicated that the herbicides at the tested concentrations did not inhibit Fusarium sp. mycelial growth, macroconidia production or germination.
\end{abstract}

Key words: mycelial growth, sporulation, germination, fungus, inhibition.

Recebido para publicação em 1.4.2002 e na forma revisada em 5.3.2004.

Parte da dissertação de Mestrado do primeiro autor apresentada à FCAV/UNESP.

${ }^{2}$ Eng.-Agr., aluno do Programa de Pós-Graduação em Produção Vegetal da Faculdade de Ciências Agrárias e Veterinárias de Jaboticabal - Universidade Estadual Paulista - FCAV/UNESP, Via de Acesso Prof. Paulo Donato Castellane, s/n. 14884-900 Jaboticabal, <mendesdiego@bol.com.br>; ${ }^{3}$ Prof. Titular, Dep. de Biologia Aplicada à Agropecuária - FCAV/UNESP, $<$ pitelli@fcav.unesp.br>; ${ }^{4}$ Eng. Florestal, Dr. Fitopatologia, pesquisador do laboratório Giorgio de Marinis - FCAV/UNESP $<$ lisias@fcav.unesp.br>. 


\section{INTRODUÇÃO}

As macrófitas são plantas muito importantes para a dinâmica dos ecossistemas de água doce, fornecendo alimentos, habitat para desova e proteção das fases jovens de organismos aquáticos; além disso, proporcionam heterogeneidade espacial, que favorece a biodiversidade do sistema. Em certas condições, algumas espécies são favorecidas, desenvolvem grandes populações e promovem uma série de interferências na dinâmica do corpo hídrico e no uso múltiplo da água. Nessas condições, as macrófitas aquáticas favorecidas são consideradas plantas daninhas e há necessidade de controle (Pietlli, 1998; Thomaz, 1998).

O gênero Egeria é nativo da América do Sul e ocorre no Brasil, no Paraguai e na Argentina. Este gênero pertence à família Hydrocharitaceae, a qual inclui as plantas aquáticas submersas de águas doce e marinha. O gênero contém somente duas espécies: E. densa e E. najas (St. John, 1961).

No Brasil, as duas espécies desse gênero causam expressivos problemas nas unidades geradoras das usinas hidrelétricas do sistema CESP (Jupiá), Light (Santana) e CHESF (Paulo Afonso). Existem grandes infestações, ainda sem problemas aparentes nas unidades geradoras de energias, em Nova Avanhandava e Promissão (Companhia Hidrelétrica do Tietê), Três Irmãos (CESP) e Salto Grande (Duke Energy). Entre todas as usinas, o problema mais sério é verificado em Jupiá, constituindo, inclusive, o motivo para a pesquisa e o desenvolvimento de Fusarium sp. (isolado FCVA\#940) como agente de controle biológico dessas duas macrófitas (Nachtigal, 2000).

No final da década de 1980 foram registradas as primeiras grandes colonizações de $E$. densa e E. najas no reservatório de Jupiá. No início da década de 1990, começaram a surgir os primeiros problemas ocasionados por essas plantas, prejudicando a pesca esportiva, a movimentação de pequenos barcos e os esportes náuticos. Pouco tempo depois, grandes massas de E. densa e E. najas começaram a ser retidas nas grades de proteção das turbinas da Usina Hidrelétrica Souza Dias, reduzindo e até impedindo o fluxo de água e, conseqüentemente, a produção de energia elétrica. Em situações extremas, as grades alteravam a forma original, ficavam comprometidas e necessitavam de substituição (Tanaka, 1998).

O manejo e o controle de populações de plantas aquáticas submersas são difíceis, devido ao crescimento profuso e à extensiva colonização dos corpos hídricos. Essas características parecem torná-las alvo ideal para a adoção de medidas de controle químico ou biológico, uma vez que o controle mecânico fica comprometido ou é inviável devido ao alto custo, à profundidade média dos reservatórios e à produção de fragmentos das plantas, que constitui a unidade básica de dispersão. No entanto, os métodos de controle químico e biológico são freqüentemente comprometidos por problemas relacionados ao equilíbrio ecológico do ambiente aquático e a aspectos técnicos, como a diluição e o tempo de contato entre o agente de controle e a planta-alvo, especialmente em condições de água corrente (Shearer, 1996).

A utilização do controle químico para plantas submersas tem custo elevado, além de representar um risco ambiental, muitas vezes, de difícil avaliação. O controle biológico destaca-se como a medida mais viável para esse tipo de vegetação, por tecnicamente apresentar menor risco ambiental. De acordo com Blackburn et al. (1971), o controle biológico constitui o meio ideal de controle de plantas daninhas aquáticas em áreas onde o uso de práticas químicas ou mecânicas é arriscado ou proibido.

Apesar de o controle de E. densa estar sendo investigado em algumas partes do mundo, especialmente nos Estados Unidos (Anderson, 1998) e no Brasil (Nachtigal \& Pitelli, 1998, 1999), pouco se conhece sobre a patologia das macrófitas submersas e não há muito conhecimento da microbiota associada às espécies em questão.

O controle de macrófitas submersas pelo uso de microrganismos, sob condições controladas, é aparentemente viável. Entretanto, a campo os resultados normalmente são inconsistentes. Segundo Charudattan et al. (1990), muitos fatores parecem contribuir para esse comportamento, como o padrão de impacto e o tempo de contato do inóculo com a planta. Para obtenção de resultados mais satisfatórios, seria interessante uma associação do controle 
químico com o biológico, uma vez que, na planta debilitada pelo contato com o herbicida, o patógeno poderia agir de forma mais rápida e efetiva.

O presente trabalho teve como objetivo avaliar o efeito de diferentes concentrações de herbicidas utilizados no controle de macrófitas aquáticas, diluídos na água, sobre o crescimento micelial, a esporulação e a germinação de macroconídios do fungo Fusarium sp. (isolado FCAV\#940).

\section{MATERIAL E MÉTODOS}

O experimento foi desenvolvido no Laboratório Giorgio de Marinis, da UNESP de Jaboticabal, no período de abril a agosto de 2001. Os herbicidas (principios ativos), em suas respectivas doses, avaliadas sob a condição de concentração diluída em uma lâmina d'água de $15 \mathrm{~cm}$, foram: Garlon $480 \mathrm{BR} \AA$ (triclopyr) a 6,0, 3,0 e 1,5 $\mathrm{mg} \mathrm{L}^{-1}$ (i.a.); Rodeo $\AA$ (glyphosate) a $6,87,3,44$ e $1,72 \mathrm{mg} \mathrm{L}^{-1}$ (i.a.); DMA $806 \mathrm{BR} \AA$ (2,4-D) a $8,54,4,27$ e $2,14 \mathrm{mg} \mathrm{L}^{-1}$ (i.a.); Reward ${ }^{\circledR}$ (diquat) a 2,0, 1,0 e 0,5 $\mathrm{mg} \mathrm{L}^{-1}$ (i.a.); e Sonar $\AA$ (fluridone) a 200, 100 e $50 \mu \mathrm{g} \mathrm{L} \mathrm{L}^{-1}$ (i.a.).

O fungo Fusarium sp. (isolado FCAV\#940), proveniente de Recife-PE (Nachtigal, 2000), obtido a partir de uma cultura-estoque constituída de solo autoclavado, foi transferido para uma placa de Petri contendo meio de cultura BDA (Batata Dextrose Ágar); esta placa foi considerada como fungo de "primeira repicagem", apresentando altíssima patogenicidade. Após o estabelecimento e o crescimento micelial do Fusarium sp. (isolado FCAV\#940) nesta placa, aproximadamente dois a três dias após a repicagem, realizou-se uma nova repicagem de um disco de $0,5 \mathrm{~cm}$ de diâmetro, contendo meio de cultura e colônia do fungo, para uma placa de Petri contendo BDA, sendo esta chamada de fungo de "segunda repicagem".

Posteriormente, após completo crescimento da colônia do fungo, aproximadamente seis dias, foram retirados discos contendo meio de cultura e colônia do fungo, os quais foram transferidos para o centro de placas de Petri contendo BDA e os respectivos herbicidas e concentrações. A incubação foi realizada em estufas de BOD a $25^{\circ} \mathrm{C}$, com fotoperíodo de 12 horas de luz fluorescente.
Para a obtenção das placas contendo as concentrações desejadas dos herbicidas, realizou-se primeiramente uma solução-estoque para cada herbicida. Para a realização da solução-estoque dos herbicidas utilizaram-se erlenmeyers de $250 \mathrm{~mL}$, sendo a calda do herbicida preparada com água destilada e estéril. Após o preparo da solução-estoque dos herbicidas, realizou-se a transferência de um volume conhecido desta solução em erlenmeyers de $250 \mathrm{~mL}$, contendo $100 \mathrm{~mL}$ de meio de cultura BDA, ainda fundente. Esse volume da solução - estoque dos herbicidas acrescentado nos $100 \mathrm{~mL}$ do meio de cultura foi previamente calculado, para se obter a concentração desejada dos herbicidas no meio de cultura. Após homogeneizar a solução meio de cultura-herbicida, verteram-se aproximadamente $20 \mathrm{~mL}$ desta solução por placa de Petri.

\section{Crescimento micelial}

O crescimento micelial do Fusarium sp. (isolado FCAV\#940), incubado em BOD a $25^{\circ} \mathrm{C}$ com fotoperíodo de 12 horas de luz fluorescente, foi acompanhado diariamente, medindose o diâmetro da colônia do fungo em dois sentidos perpendiculares entre si. A avaliação foi interrompida quando em um dos tratamentos a colônia do fungo atingiu a borda da placa de Petri.

A análise estatística dos dados foi realizada segundo esquema em parcelas subdivididas no tempo, sendo um fatorial $5 \times 4$ (cinco herbicidas e quatro concentrações) nas parcelas e cinco tempos na subparcela, em um delineamento inteiramente casualizado, com cinco repetições. Complementando a análise de variância, aplicou-se o teste de paralelismo e coincidência das retas (Mead \& Curnow, 1987); para isso, estabeleceu-se uma equação de regressão da curva de crescimento micelial $(\mathrm{cm})$, comparando-se todos os herbicidas dentro das concentrações avaliadas. Para as equações de regressão foram considerados o tempo, em dias, como variável independente e o diâmetro da colônia, em cm, como variável dependente.

\section{Esporulação}

$\mathrm{Na}$ avaliação do efeito das diferentes concentrações de herbicidas sobre a esporulação

Planta Daninha, Viçosa-MG, v.22, n.1, p.85-93, 2004 
do Fusarium sp. (isolado FCAV\#940) foram utilizadas as mesmas colônias empregadas no experimento referente ao crescimento micelial. Para isso, após a avaliação do crescimento micelial, realizou-se raspagem das colônias, empregando-se $5,0 \mathrm{~mL}$ da solução aquosa de Tween 80 a $2 \%$, mais $5,0 \mathrm{~mL}$ de água destilada estéril. Para determinar o número de conídios foi utilizado hemocitômetro, sendo as leituras realizadas em microscópio óptico. Observouse também, ao microscópio óptico, se os macroconídios do fungo apresentam-se, ou não, com alterações morfológicas.

A análise estatística dos dados foi realizada segundo o esquema fatorial $5 \times 4$ (cinco herbicidas e quatro concentrações), em um delineamento inteiramente casualizado. Complementou-se análise de variância do experimento com a utilização do teste $t$ de Student nas comparações múltiplas. Foi utilizada uma transformação logarítmica da observação dos dados [ln(observação)], para estabilizar a variabilidade dos tratamentos (Steel \& Torrie, 1960).

\section{Germinação}

Utilizou-se o meio ágar-água para a avaliação do efeito das diferentes concentrações dos herbicidas na germinação dos macroconídios de Fusarium sp. (isolado FCAV\#940) em placas contendo meio de cultura e herbicida em diferentes concentrações, sendo adotada metodologia semelhante à descrita para o item crescimento micelial. A suspensão de macroconídios do fungo, produzidos em meio BDA, sem a presença de herbicida, foi ajustada para densidade de $10^{6}$ macroconídios $\mathrm{mL}^{-1}$, a partir da qual se depositou $0,1 \mathrm{~mL}$ em placas de Petri, em três pontos predeterminados.

As placas foram incubadas em estufa de BOD mantida a $25{ }^{\circ} \mathrm{C}$, com fotoperíodo de 12 horas de luz fluorescente, sendo as 12 primeiras horas na presença de luz. Após períodos de 12, 24 e 48 horas aplicou-se azul lático, a fim de paralisar o processo de germinação e avaliar os efeitos dos herbicidas e das concentrações na germinação dos macroconídios.

A avaliação da porcentagem de germinação dos macroconídios se deu através da contagem de 100 macronídios por placa, com o auxílio de um microscópio óptico, em que se consideraram como germinados apenas os macroconídios que apresentaram o tubo germinativo maior ou igual ao tamanho do seu diâmetro. O experimento foi composto de cinco repetições, sendo cada repetição uma placa, ou seja, a contagem de 100 macroconídios.

A análise estatística foi realizada conforme descrito para o item da esporulação, porém a transformação dos dados para a análise estatística foi arco seno [raiz quadrada (proporção)], que proporcionou maior homocedasticidade dos dados.

\section{RESULTADOS E DISCUSSÃO}

\section{Crescimento micelial}

O resultado da análise de variância do crescimento micelial apresentou efeitos significativos $(\mathrm{P}<0,05)$ para os fatores herbicida e tempo $(\mathrm{P}<0,01)$ e a interação herbicida vs. tempo $(\mathrm{P}<0,01)$. Para os demais fatores e interações não se verificou efeito significativo $(\mathrm{P}>0,05)$ dos tratamentos na análise de variância. O coeficiente de determinação do modelo da análise adotada foi de $99 \%\left(\mathrm{R}^{2}=0,99\right)$, e o coeficiente de variação dos dados, de 5,1\% (CV=5,1).

Os resultados do teste de paralelismo e coincidência das retas, ilustrados nas Figuras 1 e 2 e apresentados nas Tabelas 1 e 2 , evidenciaram que a interação tripla (herbicida vs. concentração $v s$ tempo) não foi significativa no crescimento micelial de Fusarium sp. (isolado FCAV\#940) na condição de diluição das doses em uma lâmina d'água de $15 \mathrm{~cm}$. Esses resultados demonstraram que os herbicidas, em todas as concentrações avaliadas, sob a condição de volume diluído, não apresentaram efeito inibitório no crescimento micelial do agente de controle biológico, Fusarium sp. (isolado FCAV\#940).

\section{Esporulação}

Em nenhuma das concentrações dos herbicidas avaliados verificou-se deformação morfológica ou qualquer outro tipo de alteração física dos macroconídios do Fusarium sp. (isolado FCAV\#940).

O resultado da análise de variância da esporulação do fungo apresentou diferença significativa $(\mathrm{P}<0,01)$ apenas para o fator concentração. O fator herbicida e sua interação, 


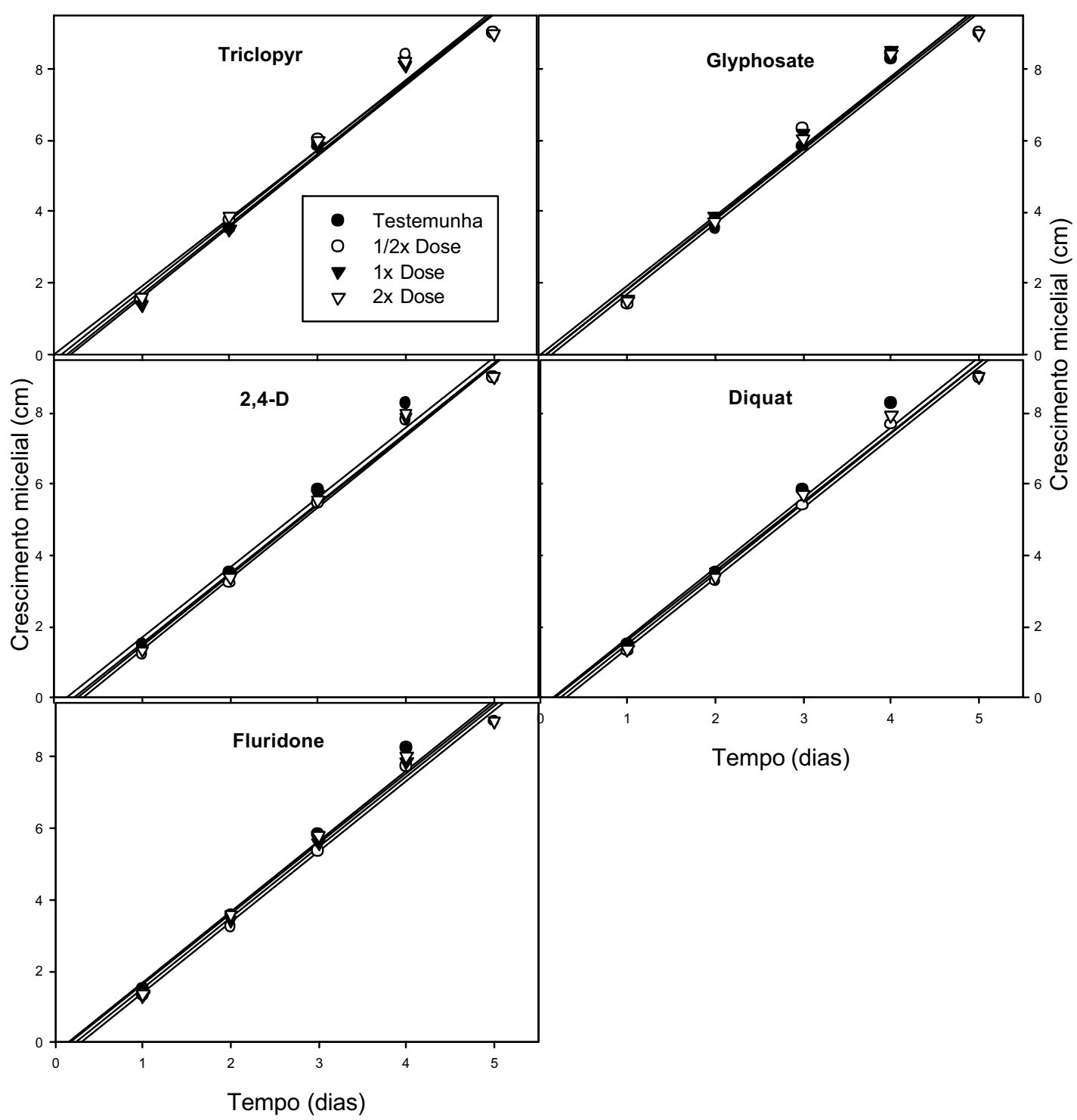

Figura 1 - Efeito de diferentes concentrações de cinco herbicidas adicionados em meio BDA no crescimento de colônia de Fusarium sp. (isolado FCAV\#940), após cinco dias de incubação em BOD a $25^{\circ} \mathrm{C}$ e fotoperíodo de 12 horas.

herbicida vs. concentração, não apresentaram diferença significativa $(\mathrm{P}>0,05)$. O coeficiente de determinação do modelo da análise adotada foi de $29 \%\left(R^{2}=0,29\right)$, e o coeficiente de variação dos dados, de 4,33\% (CV=4,33).

Com relação às concentrações avaliadas, observou-se que a testemunha (BDA sem herbicida) apresentou melhor média de esporulação, a qual diferiu estatisticamente das demais concentrações estuda das (Tabela 3).
Apesar de não ter sido observado diferença estatística entre os herbicidas, verificou-se tendência de menor produção de esporos quando o fungo foi submetido aos tratamentos com os herbicidas 2,4-D e diquat. No entanto, o herbicida glyphosate foi o único tratamento que ocasionou aumento no número de esporos do fungo quando comparado com a testemunha, sendo observado maior número de esporos na condição de dose recomendada, seguido da condição de metade da dose (Tabela 3).

Planta Daninha, Viçosa-MG, v.22, n.1, p.85-93, 2004 


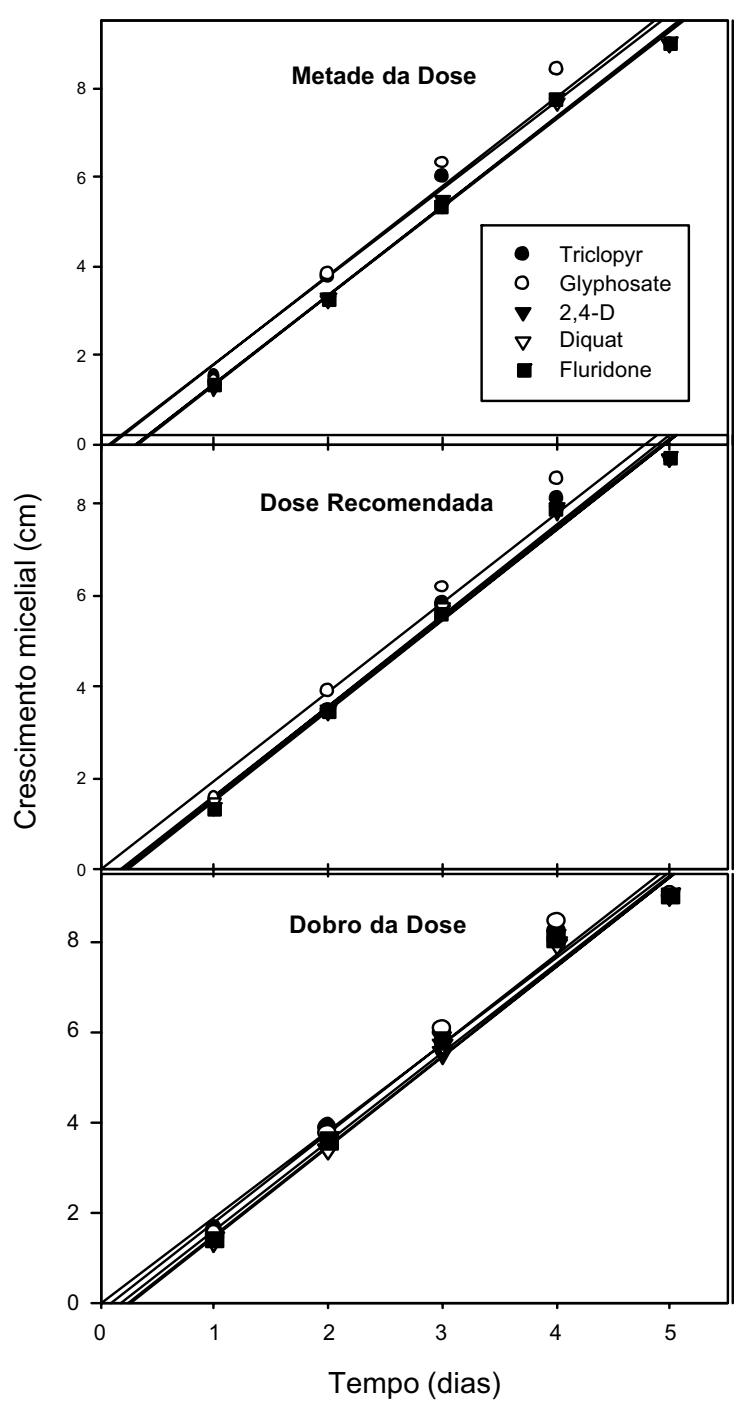

Figura 2 - Efeito dos diferentes herbicidas, nas três concentrações avaliadas, adicionados em meio BDA no crescimento de colônia de Fusarium sp. (isolado FCAV\#940), após cinco dias de incubação em BOD a $25^{\circ} \mathrm{C}$ e fotoperíodo de 12 horas.

\section{Germinação}

O resultado da análise de variância apresentou efeito estatisticamente significativo $(\mathrm{P}<0,05)$ para o fator herbicida e a interação herbicida vs. concentração. Entretanto, não foi detectado efeito significativo $(P>0,05)$ para 0 fator concentração. O coeficiente de determinação para o modelo da análise dos dados foi de $35 \%\left(R^{2}=0,35\right)$, e o coeficiente de variação, de $3,73 \%(C V=3,73)$.

A germinação dos macroconídios de Fusarium sp. (isolado FCAV\#940) em meio de cultura ágar-água, contendo as concentrações dos herbicidas avaliados, após 12 horas de incubação em BOB a $25{ }^{\circ} \mathrm{C}$ com luz constante, apresentou médias variando de 86,6 a $93,8 \%$ de germinação dos macroconídios do fungo. Esses valores encontrados de porcentagem de germinação, nesse período de tempo, do ponto de vista fitopatológico, foi bastante satisfatório.

Para os herbicidas triclopyr e glyphosate, verificou-se que a variação das concentrações testadas na germinação dos macroconídios do fungo não foram estatisticamente diferentes entre si.

Foi possível observar que o herbicida glyphosate foi o único que apresentou germinação superior à da testemunha em todas as concentrações testadas. Constatou-se que os herbicidas triclopyr e diquat apresentaram diminuição da germinação, independentemente da concentração avaliada, quando comparado com a testemunha (Tabela 4).

Devido ao fato de o fungo ter apresentado uma média de germinação satisfatória logo nas primeiras 12 horas, não se avaliou a germinação nas horas seguintes, pois em muitos dos tratamentos, no tempo de 24 horas, observouse crescimento micelial intenso, havendo, em alguns casos, esporulação na placa e dificuldade na leitura da germinação, o que ocorreu para todos os tratamentos na avaliação da germinação em 48 horas. Esses resultados demonstraram o potencial da utilização do fungo em associação com qualquer um dos herbicidas avaliados.

Pelas condições do presente estudo, concluiu-se que os herbicidas avaliados, em qualquer uma das doses testadas, sob a condição de diluição em lâmina d'água de $15 \mathrm{~cm}$ não apresentaram efeito inibitório no crescimento micelial, na esporulação e na germinação dos macroconídios de Fusarium sp. (isolado FCAV\#940). A associação deste fungo com qualquer um dos herbicidas estudados poderia ser uma alternativa para otimizar o controle das macrófitas aquáticas Egeria najas e E. densa. 
Tabela 1 - Equações de regressão do crescimento micelial de Fusarium sp. (isolado FCAV\#940) em função do tempo das diferentes concentrações dos cinco herbicidas adicionados em meio BDA e incubados por período de cinco dias em BOD a $25{ }^{\circ} \mathrm{C}$ e fotoperíodo de 12 horas

\begin{tabular}{|c|c|c|c|c|c|c|c|c|c|c|}
\hline \multirow{2}{*}{ Concentração } & \multirow{2}{*}{ Equação de regressão } & \multirow{2}{*}{$\mathrm{r}$} & \multicolumn{2}{|c|}{$\mathrm{r} 1$} & \multicolumn{2}{|c|}{ r2 } & \multicolumn{2}{|c|}{ r3 } & \multicolumn{2}{|c|}{$\mathrm{r} 4$} \\
\hline & & & $\mathrm{C}$ & $\mathrm{P}$ & $\mathrm{C}$ & $\mathrm{P}$ & $\mathrm{C}$ & $\mathrm{P}$ & $\mathrm{C}$ & $\mathrm{P}$ \\
\hline \multicolumn{11}{|c|}{ Triclopyr } \\
\hline $\begin{array}{l}\text { Test. }(0 \mathrm{ppm})(\mathrm{r} 1) \\
\text { Metade da dose }(\mathrm{r} 2)\end{array}$ & $\begin{array}{l}y=-0,29+1,97 x \\
y=-0,15+1,96 x\end{array}$ & $\begin{array}{l}0,9891 * * \\
0,9862 * *\end{array}$ & $\begin{array}{l}- \\
-\end{array}$ & - & ns & $\mathrm{ns}$ & $\begin{array}{ll}\mathrm{ns} \\
\mathrm{ns}\end{array}$ & $\begin{array}{l}\mathrm{ns} \\
\mathrm{ns}\end{array}$ & $\begin{array}{l}\mathrm{ns} \\
\mathrm{ns}\end{array}$ & $\begin{array}{l}\mathrm{ns} \\
\mathrm{ns} \\
\end{array}$ \\
\hline $\begin{array}{l}\text { Dose recomendada (r3) } \\
\text { Dobro da dose (r4) }\end{array}$ & $\begin{array}{l}y=-0,39+1,99 x \\
y=-0,01+1,92 x\end{array}$ & $\begin{array}{l}0,9909 * * \\
0,9893 * *\end{array}$ & - & - & - & - & - & - & ns & ns \\
\hline \multicolumn{11}{|c|}{ Glyphosate } \\
\hline $\begin{array}{l}\text { Test. (0 ppm) (r1) } \\
\text { Metade da dose (r2) }\end{array}$ & $\begin{array}{l}y=-0,29+1,97 x \\
y=-0,16+1,98 x\end{array}$ & $\begin{array}{l}0,9891 * * \\
0,9819 * *\end{array}$ & $\begin{array}{l}- \\
-\end{array}$ & - & ns & ns & $\begin{array}{l}\mathrm{ns} \\
\mathrm{ns}\end{array}$ & $\begin{array}{l}\mathrm{ns} \\
\mathrm{ns}\end{array}$ & $\begin{array}{l}\mathrm{ns} \\
\mathrm{ns}\end{array}$ & $\begin{array}{l}\mathrm{ns} \\
\mathrm{ns}\end{array}$ \\
\hline $\begin{array}{l}\text { Dose recomendada (r3) } \\
\text { Dobro da dose (r4) }\end{array}$ & $\begin{array}{l}y=-0,01+1,95 x \\
y=-0,16+1.97 x\end{array}$ & $\begin{array}{l}0,9826 * * \\
0.9854 * *\end{array}$ & - & - & - & - & - & - & ns & $\mathrm{ns}$ \\
\hline \multicolumn{11}{|c|}{ 2,4-D } \\
\hline Test. $(0$ ppm $)(\mathrm{r} 1)$ & $y=-0,29+1,97 x$ & $0,9891 * *$ & - & - & ns & $\mathrm{ns}$ & ns & $\mathrm{ns}$ & $\mathrm{ns}$ & ns \\
\hline Metade da dose (r2) & $y=-0,67+2,01 x$ & $0,9957 * *$ & - & - & - & $=$ & ns & ns & ns & ns \\
\hline Dose recomendada (r3) & $y=-0,43+1,97 x$ & $0,9946 * *$ & - & - & - & $=$ & - & - & ns & ns. \\
\hline Dobro da dose $(\mathrm{r} 4)$ & $y=-0,52+1,99 x$ & $0,9939 * *$ & - & - & - & - & - & - & & - \\
\hline \multicolumn{11}{|c|}{ Diquat } \\
\hline Test. $(0$ ppgm $2(r 1)$ & $\mathrm{y}=-0.29+1.97 \mathrm{x}$ & $0.9891 * *$ & - & - & nns. & nns & ns. & -nns. & ns. & -ns. \\
\hline Metade da dose (r2) & $y=-0,61+1,98 x$ & $0,9972 * *$ & - & - & - & - & ns & $\mathrm{ns}$ & ns & ns \\
\hline Dose recomendada (r3) & $y=-0,33+1,96 x$ & $0,9937 * *$ & - & - & - & - & - & - & ns & ns \\
\hline Dobro da dose (r4) & $y=-0,46+1,98 x$ & $0,9938 * *$ & - & - & - & - & - & - & & \\
\hline \multicolumn{11}{|c|}{ Fluridone } \\
\hline Test. (0pp & $\mathrm{Y}=-0.29+1,97 \mathrm{x}$ & $0.9891 * *$ & - & - & ns & nns & ns. & nns. & ns. & ns. \\
\hline Metade da dose (r2) & $\mathrm{Y}=-0,60+1,98 \mathrm{x}$ & $0,9966 * *$ & - & - & -1 & & ns & ns & ns & ns \\
\hline Dosese recomemendada $(\mathrm{r} 3)$ & $\mathrm{Y}=-0,49+1,98 \mathrm{x}$ & $0.9947 * *$ & - & - & & - & - & - & ns. & \\
\hline Dobro da dose (r4) & $Y=-0,36+1,97 x$ & $0,9921 * *$ & - & - & - & - & - & - & & \\
\hline
\end{tabular}

$\mathrm{r}=$ coeficiente de correlação; $\mathrm{C}=$ coincidência das retas pelo teste $\mathrm{F} ; \mathrm{P}=$ paralelismo das retas pelo teste $\mathrm{t}$; ns = não-significativo.

Tabela 2 - Equações de regressão do crescimento micelial de Fusarium sp. (isolado FCAV\#940) em função do tempo das três diferentes concentrações dos herbicidas avaliados em meio BDA e incubados por um período de cinco dias em BOD a $25{ }^{\circ} \mathrm{C}$ e fotoperíodo de 12 horas

\begin{tabular}{|c|c|c|c|c|c|c|c|c|c|c|c|c|}
\hline \multirow{2}{*}{ Herbicida } & \multirow{2}{*}{ Equação de regressão } & \multirow{2}{*}{$\mathrm{r}$} & \multicolumn{2}{|c|}{$\mathrm{r} 1$} & \multicolumn{2}{|c|}{$\mathrm{r} 2$} & \multicolumn{2}{|c|}{ r3 } & \multicolumn{2}{|c|}{$\mathrm{r} 4$} & \multicolumn{2}{|c|}{ r5 } \\
\hline & & & $\mathrm{C}$ & $\mathrm{P}$ & $\mathrm{C}$ & $\mathrm{P}$ & $\mathrm{C}$ & $\mathrm{P}$ & $\mathrm{C}$ & $\mathrm{P}$ & $\mathrm{C}$ & $\mathrm{P}$ \\
\hline \multicolumn{13}{|c|}{ Metade da dose } \\
\hline $\begin{array}{l}\text { Triclopyr (r1) } \\
\text { Glyphosate (r2) }\end{array}$ & $\begin{array}{l}y=-0,15+1,96 \cdot x \\
y=-0,16+1,98 \cdot x\end{array}$ & $\begin{array}{l}0,9862 * * \\
0,9819 * *\end{array}$ & $\begin{array}{l}- \\
- \\
-\end{array}$ & - & ns & ns & $\begin{array}{r}\mathrm{ns} \\
\mathrm{ns}\end{array}$ & $\begin{array}{l}\mathrm{ns} \\
\mathrm{n} \mathrm{n}\end{array}$ & $\begin{array}{l}\text { ns } \\
\text { ns }\end{array}$ & $\begin{array}{l}\text { ns } \\
\text { ns }\end{array}$ & $\begin{array}{l}\mathrm{ns} \\
\mathrm{ns}\end{array}$ & $\begin{array}{l}\mathrm{ns} \\
\mathrm{ns} \text {. }\end{array}$ \\
\hline $2,4-\mathrm{D}(\mathrm{r} 3)$ & $y=-0,67+2,01 . x$ & $0,9957 * *$ & - & - & - & - & - & - & $\mathrm{ns}$ & ns & $\mathrm{ns}$ & ns \\
\hline . Diqụaț (r4). & $y=-0,61+1,98 \cdot x$. & $0.9972 * *$ & $=$ & - & - & 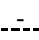 & & 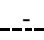 & & & ns & . nss. \\
\hline Fluridone (r5) & $y=-0,60+1,98 . x$ & $0,9966 * *$ & - & - & - & - & - & - & - & - & - & $\begin{array}{ll}-1 \\
-1\end{array}$ \\
\hline \multicolumn{13}{|c|}{ Dose recomendada } \\
\hline Triclopyr $(\mathrm{r} 1)$ & $y=-0,39+1,99 \cdot x$ & $0.9909 * * .$. & $\ldots$ & $=$ & ns & ns & nns & _ns & ns & ns & ns & ns. \\
\hline Glyphosate (r2) & $y=-0,01+1,95 \cdot x$ & $0.9826 * *$ & - & $=$ & - & - & ns & n- & ns & ns & ns & ns. \\
\hline $2,4-\mathrm{D}(\mathrm{r} 3)$ & $y=-0,43+1,97 \cdot x$ & $0,9946 * *$ & - & - & - & - & - & - & ns & ns & ns & ns. \\
\hline . Diquat (r4) & $y=-0,33+1,96 \times x$ & $0.9937, \ldots *$ & - & $=$ & $=$ & $=$ & $=$ & - & - & - & ns & ns. \\
\hline Fluridone (r5) & $y=-0,49+1,98 \cdot x$ & $0,9947 * *$ & - & - & - & - & - & - & - & - & - & - \\
\hline \multicolumn{13}{|c|}{ Dobro da dose } \\
\hline Triclopyr $(\mathrm{r} 1)$ & $y=-0,01+1,92 \cdot x$ & $0.9893 * *$ & $=$ & - & ns & $n \mathrm{n}$ & nns & nns & ns & ns & ns & ns. \\
\hline Glyphosate (r2) & $y=-0,16+1,97 \cdot x$ & $0,9854 * *$ & - & - & - & - & ns & $\mathrm{ns}$ & ns & ns & $\mathrm{ns}$ & ns \\
\hline $2,4-\mathrm{D}(\mathrm{r} 3)$ & $y=-0,52+1,99 . x$ & $0.9939 * *$ & & - & - & - & $=$ & - & nns & nss & ns. & ns. \\
\hline Diquat (r4) & $y=-0,46+1,98 \cdot x$ & $0,9938 * *$ & - & - & $=$ & - & - & - & - & - & ns & ns. \\
\hline Fluridone (r5) & $y=-0.36+1,97 . x$ & $0.9921 * *$ & - & - & - & - & - & - & - & - & - & - \\
\hline
\end{tabular}

$\mathrm{r}=$ coeficiente de correlação; $\mathrm{C}=$ coincidência das retas pelo teste $\mathrm{F} ; \mathrm{P}=$ paralelismo das retas pelo teste $\mathrm{t}$; ns = não-significativo. 
Tabela 3 - Esporulação de Fusarium sp. (isolado FCAV\#940) resultante do efeito de concentrações de cinco herbicidas adicionados em meio BDA, incubados durante cinco dias em BOD a $25^{\circ} \mathrm{C}$ e fotoperíodo de 12 horas

\begin{tabular}{|c|c|c|c|c|c|c|}
\hline \multirow{2}{*}{ Concentração } & \multicolumn{5}{|c|}{ Herbicida } & \multirow{2}{*}{ Média } \\
\hline & Triclopyr & Glyphosate & 2,4-D & Diquat & Fluridone & \\
\hline Test. (0 ppm) & 1.265 .000 & 1.265 .000 & 1.265 .000 & 1.265 .000 & 1.265 .000 & $1.265 .000 \mathrm{a}$ \\
\hline Metade da dose & 880.000 & 1.295 .000 & 500.000 & 1.190 .000 & 1.070 .000 & $987.000 \mathrm{~b}$ \\
\hline Dose tecomendada & 1.035 .000 & 1.385 .000 & 690.000 & 585.000 & 660.000 & $871.000 \mathrm{~b}$ \\
\hline Dobro da dose & 1.025 .000 & 1.020 .000 & 990.000 & 710.000 & 870.000 & $923.000 \mathrm{~b}$ \\
\hline Média & $1.051 .250 \mathrm{~A}$ & $1.241 .250 \mathrm{~A}$ & $861.250 \mathrm{~A}$ & $937.500 \mathrm{~A}$ & $966.250 \mathrm{~A}$ & - \\
\hline
\end{tabular}

Análise estatística realizada com dados transformados em ln da observação, porém os valores das médias apresentadas são originais.

Médias seguidas da mesma letra, minúscula na coluna e maiúscula na linha, não diferem entre si pelo teste $t$ de Student a $5 \%$ de probabilidade.

Tabela 4 - Porcentagem de germinação de macroconídios de Fusarium sp. (isolado FCAV\#940), em meio de cultura ágar-água com concentrações crescentes de diferentes herbicidas após 12 horas de incubação em BOD a $25^{\circ} \mathrm{C}$ na presença de luz fluorescente

\begin{tabular}{|l|c|c|c|c|c|c|}
\hline \multirow{2}{*}{ Concentração } & \multicolumn{5}{|c|}{ Herbicida } & \multirow{2}{*}{ Média } \\
\cline { 2 - 7 } & Triclopyr & Glyphosate & $2,4-\mathrm{D}$ & Diquat & Fluridone & \\
\hline Test. (0 ppm) & $91,0 \mathrm{Aa}$ & $91,0 \mathrm{Aa}$ & $91,0 \mathrm{Abc}$ & $91,0 \mathrm{Aa}$ & $91,0 \mathrm{Aab}$ & $91,0 \mathrm{a}$ \\
\hline Metade da dose & $9 . \ldots, 0 \mathrm{ABa}$ & $93,0 \mathrm{Aa}$ & $88,4 \mathrm{Bc}$ & $88,6 \mathrm{Ba}$ & $88,6 \mathrm{Bbc}$ & $89,72 \mathrm{a}$ \\
Dose cheia & $89,4 \mathrm{BCa}$ & $91,4 \mathrm{ABa}$ & $93,8 \mathrm{Aa}$ & $87,6 \mathrm{Ca}$ & $92,6 \mathrm{Aa}$ & $90,96 \mathrm{a}$ \\
Dobro da dose & $89,6 \mathrm{ABa}$ & $90,4 \mathrm{ABa}$ & $92,2 \mathrm{Aab}$ & $89,4 \mathrm{Aba}$ & $86,6 \mathrm{Bc}$ & $89,64 \mathrm{a}$ \\
\hline Média & $90 \mathrm{AB}$ & $91,45 \mathrm{~A}$ & $91,35 \mathrm{~A}$ & $89,15 \mathrm{~B}$ & $89,7 \mathrm{AB}$ & - \\
\hline
\end{tabular}

Análise estatística realizada com dados transformados em arco seno $\left(V_{\mathrm{X}} / 100\right)$, porém os valores das porcentagens médias apresentadas são originais.

Médias seguidas da mesma letra, minúscula na coluna e maiúscula na linha, não diferem entre si pelo teste de $\mathrm{t}$ de Student a $5 \%$ de probabilidade.

\section{AGRADECIMENTOS}

À CAPES, pela bolsa concedida; ao Prof. Dr. Gener Tadeu Pereira, pelo auxílio nas análises estatísticas dos dados; ao Prof. Dr. Euclides Braga Malheiros e Prof. Dr. Antonio de Góes, pelas sugestões oferecidas.

\section{LITERATURA CITADA}

ANDERSON, L. W. J. Can Egeria densa be eradicated? Yes, but is it worth it?. In: ANNUAL MEETING OF AQUATIC PLANT MANAGEMENT SOCIETY, 38., 1998, Memphis. Abstracts... Memphis: 1998. p. 18.

BLACKBURN, R. D.; SUTTON, D. L.; TAYLOR, T. Biological control of aquatic weeds. J. Irrig. Drain., v. 97, n. 3, p. 421-432, 1971

CHARUDATTAN, R.; DE VALERIO, J. T.; PRANGE, V. J. Special problems associated with aquatic weed control. In: BAKER, R.; DUNN, P. E. New directions in biological control: alternatives for suppressing agricultural pests and diseases. New York: Alan R. Liss, 1990. p. 287-303.
MEAD, R.; CURNOW, R. N. Statistical methods in agriculture and experimental biology. London: Chapman and Hall, 1987. 335 p.

NACHTIGAL, G. F. Desenvolvimento de agente de controle biológico microbiano de Egeria densa e Egeria najas. 2000. $160 \mathrm{f}$. Tese (Doutorado em Produção Vegetal) - Universidade Estadual Paulista, Jaboticabal, 2000.

NACHTIGAL, G. F.; PITELLI, R. A. Fusarium sp. as a potential biocontrol agent for Egeria densa and Egeria najas. In: INTERNATIONAL SYMPOSIUM ON BIOLOGICAL CONTROL OF WEEDS, 10., 1999, Montana, Bozeman. Anais... Montona, Bozeman: 1999. p. 68.

NACHTIGAL, G. F.; PITELLI, R. A. Fusarium sp. e seu potencial como agente de controle biológico de Egeria densa e Egeria najas no Brasil. In: SICONBIOL, 5., 1998, Rio de Janeiro. Anais... Rio de Janeiro: 1998. p. 140 . 
PITELLI, R. A. Macrófitas aquáticas no Brasil, na condição de problemáticas. In: WORKSHOP CONTROLE DE PLANTAS AQUÁTICAS, 1998, Brasília. Resumos... Brasília: IBAMA, 1998. p. 12-15.

SHEARER, J. F. Development of a fungal pathogen for biocontrol of the submersed aquatic macrophyte Hydrilla verticillata. In: INTERNATIONAL SYMPOSIUM ON BIOLOGICAL CONTROL OF WEEDS, 9., 1996, Stellenbosch. Proceedings... Stellenbosch: 1996. p. $473-477$.

ST. JOHN, H. Monograph of the genus Egeria Planchon Darwiniana, v. 12, n. 2, p. 293-307, 1961
STEEL, R. G. D.; TORRIE, J. H. Principles and procedures of statistics: with special reference to the biological sciences. New York: Mc Graw-Hill Book, 1960. $481 \mathrm{p}$.

TANAKA, R. H. Prejuízos provocados pelas plantas aquáticas. In: WORKSHOP SOBRE CONTROLE DE PLANTAS AQUÁTICAS, 1998, Brasília. Resumos... Brasília: IBAMA, 1998. p. 36-38.

THOMAZ, S. M. Explosões populacionais de plantas aquáticas: sintoma de um problema. In: WORKSHOP SOBRE CONTROLE DE PLANTAS AQUÁTICAS, 1998, Brasília. Resumos... Brasília: IBAMA, 1998. p. 16-17. 